\title{
Characterization of Cider Apple Fruits According to Their Degree of Ripening. A Chemometric Approach
}

\author{
J uan J . Mangas, ${ }^{* \dagger}{ }^{\dagger}$ J avier Moreno, ${ }^{\dagger}$ Anna Picinelli, ${ }^{\dagger}$ and Domingo Blanco \\ Centro de Investigación Aplicada y Tecnología Agroalimentaria, Apartado 13, E-33300 Villaviciosa, Spain, \\ and Departamento de Química Física y Analítica, Facultad de Química, Universidad de Oviedo, \\ E-33006 Oviedo, Spain
}

\begin{abstract}
A chemometric study was carried out in order to typify cider apples according to their degree of ripening. Several chemical variables (sugars, organic acids, amino acids, polyphenols, and pectins) were analyzed using HPLC and FIA methods. Univariate data treatment was not sufficient to allow the apple varieties to be differentiated according to their stage of ripening. Two linear combinations of original variables, ascertained by principal component analysis (PCA), provided an adequate data structurization. To classify apples by their degree of ripening, a mathematical decision rule was established with a prediction capacity of $85 \%$ using a LDA method; the most relevant variables in the canonical function ascertained by LDA were sugars, pectins, malic acid, glycine, serine, valine, and glutamic acid. The use of the PLS-2 al gorithm demonstrated the influence of the ripening process on the chemical composition of the fruits $\left(R^{2}: 91.7 \%\right)$ and furthermore allowed authors to differentiate apple varieties according to their degree of ripening.
\end{abstract}

Keywords: Apple; ripening; chemometric

\section{INTRODUCTION}

For practical reasons, cider apples should be biotransformed when certain technological quality criteria have been satisfied. However, establishing these criteria is a difficult task since many factors can influence the apple biotransformation process in cidermaking. For instance, sugars and organic acids are typical substrates metabolized by yeasts and bacteria during apple juice biotransformation by means of the alcoholic and malolactic pathways. Amino acids are the assimilable nitrogen fraction, being furthermore the precursors of certain aroma components such as higher alcohols (Henschke and J iranek, 1993). Polyphenols are products of the secondary metabolism of plants which not only affect the taste and flavor of cider (Lea, 1995) but also control the metabolism of microorganisms. As is well-known, the PPO system depletes the oxygen level due to the oxidation of polyphenols which affects microorganism equilibria, and hence the aroma of cider may be significantly modified. Pectins are structural polysaccharides which control apple fruit softening; from a technol ogical viewpoint, pectins are closely related to the clarification process (Le Quere et al., 1988) and cider stabilization.

A number of studies for establishing the ontogenic stage for technological development purposes in apple fruit have been carried out in recent years. Blanco et al . (1992a,b), Ackermann et al. (1992), Berüter (1989), and Mangas et al. (1992) detected significant changes in the apple composition in the final steps of ripening; for instance, an accumulation of sugars and pectins (water-soluble pectin and chelator-soluble pectin) was

\footnotetext{
* To whom correspondence should be addressed.

+ Centro de Investigación Aplicada y Tecnología Agroalimentaria.

‡ Universidad de Oviedo.
}

observed, while at the same time, a decrease in organic acids and certain amino acids, such as asparagine, was also detected. Likewise, the concentration of total polyphenols and nitrogen in cider apples reaches a minimum prior to the final accumulation of sugars and starch degradation (Blanco et al., 1992a).

Since the evolution of the apple fruit during its senescence is determined by different biochemical processes which are interrel ated, several chemical markers are needed to correctly typify the degree of ripening of the cider apple; multivariate treatment of data is therefore essential in order to achieve this purpose. Chemometrics have been extensively used in this sense, for characterizing fruits (Fils-Lycaon et al., 1988; McRae et al., 1990) and alcoholic beverages (Cruz Ortiz et al., 1993; Mangas et al., 1996, 1997).

Traditionally, the optimum ripening stage of apple ciders used for cidermaking has been evaluated by the apple starch content and the total sugars/total acidity ratio. However, other substances such as organic acids, polyalcohols, individual sugars, amino acids, pectins, and polyphenols, that notably influence cider quality, should also be taken in account for determining the stage of ripening of apples that are used for biotransformation purposes.

The aim of the present study was to use various chemical markers of fruit ripening, cl osely related to the quality of processed apple products, in conjunction with chemometrics, to ascertain a general prediction rule for the stage of ripening of a number of apple cider cultivars selected because of their technological suitability for cidermaking.

\section{MATERIALS AND METHODS}

Samples. Six Spanish cider apple cultivars, Collaos (mild sharp), Durón Arroes and Picona Rayada (sweet), Meana (bittersharp), Raxao (sharp), and Coloradona (bittersweet) 
were picked (each picking level was associated with a harvest date) during two harvests, from September to December, so as to carry out ripening studies; sampling was carried out initally every 15 days and, subsequently, in the final stage of ripening, every 5 days. Sample preparation for analyses was carried out according to the methodology described by Blanco et al. (1992a) using a modified version of Richmond's method (Richmond et al., 1981). Fresh apples (1 kg; 8-16 fruits) were harvested, and $250 \mathrm{~g}$ was directly sampled and placed in a blender with a volume of ethanol and hydrochloric acid in such a way that $80 \%(\mathrm{w} / \mathrm{w})$ and $0.1 \%(\mathrm{v} / \mathrm{v})$ was obtained, respectively. The fruits and solvents were then blended at high speed for $2 \mathrm{~min}$. The resulting slurry was refluxed under a nitrogen atmosphere for $2 \mathrm{~h}$. The extract obtained was subjected to a concentration process under vacuum, and once the final volume had been adjusted with $80 \%(\mathrm{v} / \mathrm{v})$ ethanol, the resulting extract was microfiltered prior to chromatographic and FIA analyses. Sample preparation for pectin analysis was conducted according to the method described by Mangas et al. (1992).

Analytical Procedures. Sugars (sucrose, glucose, fructose and sorbitol), organic acids (quinic, malic and shikimic), and amino acids (aspartic, glutamic, asparagine, serine, glutamine, glycine, arginine, $\beta$-alanine, alanine, valine, phenylalanine and leucine) were analyzed by HPLC using the methodologies described by Blanco et al. (1988a,b, 1990), respectively. A Waters chromatographic system was employed for sugars analysis with the following makeup: Sugar Pak I column (300 $\times 6.5 \mathrm{~mm}$ ); mobile phase, $50 \mathrm{mg} / \mathrm{L}$ of the calcium salt of EDTA; flow, $0.5 \mathrm{~mL} / \mathrm{min}$; column temperature, $80{ }^{\circ} \mathrm{C}$; detection, refractive index. A LKB chromatograph was used for the analyses of organic acids and amino acids; organic acids: Spherisorb ODS-2 col umn $(250 \times 4 \mathrm{~mm} ; 5 \mu \mathrm{m})$; mobile phase, $0.01 \mathrm{M}$ potassium dihydrogen phosphate buffer adjusted to $\mathrm{pH}$ 2.25; flow, $0.5 \mathrm{~mL} / \mathrm{min}$; process temperature, $25{ }^{\circ} \mathrm{C}$; UV detection at $206 \mathrm{~nm}$; amino acids: Spherisorb ODS-2 column $(300 \times 4.6 \mathrm{~mm} ; 5 \mu \mathrm{m})$ and guard column packed with the same material $(40 \mu \mathrm{m})$; gradient elution [solvent A, $0.01 \mathrm{M}$ dihydrogen orthophosphate ( $\mathrm{pH} \mathrm{6.8)}$ with sodium nitrate up to 0.08 $M$ of ionic strength and $1 \%$ tetrahydrofuran; solvent $B$, methanol ], $3 \%$ B for $1 \mathrm{~min}$, linear step at $15 \%$ B for $1 \mathrm{~min}$, isocratic for $8 \mathrm{~min}$, linear step at $25 \%$ B for $5 \mathrm{~min}$, isocratic for $5 \mathrm{~min}$, linear step to $50 \%$ B for $5 \mathrm{~min}$, isocratic for $5 \mathrm{~min}$, linear step at $100 \% \mathrm{~B}$ for $15 \mathrm{~min}$ and isocratic for $10 \mathrm{~min}$; column temperature, $30{ }^{\circ} \mathrm{C}$; flow, $1.3 \mathrm{~mL} / \mathrm{min}$; derivatizing solution, o-phthaldialdehyde/2-mercaptoethanol; OPA derivatives were monitored at an excitation wavelength of $340 \mathrm{~nm}$ and an emission wavelength of $425 \mathrm{~nm}$. Pectins [water-soluble pectin (WSP), chelator-soluble pectin (CSP) and hydrochloric acid-soluble pectin (HASP)] were determined by spectrophotometry and measured as total galacturonic acid according to the method described by Kintner and Van Buren (1982) and Thibault (1979) by incubation with sulfuric acid and subsequent color development with alkaline m-hydroxydiphenyl. Total polyphenols were analyzed using a FIA system according to the method optimized by Mangas et al. (1993). To do this, we used the Folin-Ciocalteu reagent and an FIA system with the following makeup: carrier, Folin-Ciocalteu reagent (1/ 100) with $\mathrm{Na}_{2} \mathrm{CO}_{3} 1 \%(\mathrm{w} / \mathrm{v})$ at a flow of $0.2 \mathrm{~mL} / \mathrm{min}$; reagent, $\mathrm{Na}_{2} \mathrm{CO}_{3} 1 \%$ at a flow of $0.6 \mathrm{~mL} / \mathrm{min}$; two coils $(300 \times 0.5 \mathrm{~mm}$; $300 \times 0.7 \mathrm{~mm}$ ); colorimetric detection at $673 \mathrm{~nm}$; injection volume $40 \mu \mathrm{L}$.

Data Processing. The PARVUS statistical package (F orina et al., 1988) was used for data processing. A data matrix was constructed with rows (80) representing apples with different degree of ripening, and columns (23) corresponding to chemical variables (sugars, organic acids, pectins, polyphenols, and amino acids) monitored during the ripening process of the apples. Samples were initially categorized as ripe (R, $31)$ and unripe $(U, 49)$ on the basis of the starch content and the total sugar/total acidity ratio (Blanco et al., 1992a). Several multivariate statistical techniques were employed in order to visualize the data structure and to typify apples according to their degree of ripening, namely Principal Component Analysis (PCA), Linear Discriminant Analysis (LDA),
Table 1. Descriptive Statistics of Variables $(n=80)^{a}$

\begin{tabular}{lrrrrrrr}
\hline variable & $\begin{array}{r}\text { mean } \\
(\mathrm{g} / \mathrm{kg})\end{array}$ & \multicolumn{1}{c}{ SD } & \multicolumn{6}{c}{ skewness } & kurtosis & min. & max. & range \\
\hline S & 18.76 & 10.66 & 1.37 & 1.50 & 5.95 & 52.30 & 46.35 \\
G & 13.41 & 5.04 & 0.27 & -0.81 & 5.27 & 26.54 & 21.27 \\
F & 44.54 & 9.40 & 0.76 & 0.59 & 28.81 & 75.03 & 46.22 \\
So & 5.53 & 2.65 & 1.36 & 1.85 & 1.78 & 14.44 & 12.66 \\
P & 3.91 & 1.41 & 0.99 & 0.54 & 1.87 & 8.66 & 6.79 \\
WSP & 1.48 & 1.57 & 2.74 & 12.63 & 0.03 & 10.31 & 10.28 \\
CSP & 1.63 & 1.44 & 0.97 & 0.66 & 0.08 & 6.59 & 6.51 \\
HASP & 19.18 & 11.59 & 0.39 & -0.26 & 2.45 & 51.88 & 49.43 \\
Q & 0.43 & 0.39 & 2.36 & 7.50 & 0.02 & 2.35 & 2.33 \\
M & 5.67 & 2.90 & 0.41 & -0.66 & 1.28 & 13.79 & 12.51 \\
Sh* & 24.24 & 13.02 & 0.48 & -0.70 & 6.20 & 52.70 & 46.50 \\
Asp & 83.90 & 37.84 & 0.30 & -0.04 & 4.04 & 195.70 & 191.66 \\
Glu & 90.45 & 47.71 & 0.78 & 0.54 & 12.90 & 218.20 & 205.30 \\
Asn & 378.94 & 350.50 & 3.07 & 12.44 & 38.90 & 2087.50 & 2048.60 \\
Ser & 27.67 & 12.82 & 2.10 & 6.49 & 6.70 & 86.40 & 79.70 \\
Gln & 15.88 & 20.91 & 5.99 & 43.54 & 0.10 & 175.10 & 175.00 \\
Gly & 6.79 & 3.93 & 1.00 & 1.02 & 0.40 & 20.60 & 20.20 \\
Arg & 5.81 & 3.19 & 1.09 & 0.61 & 1.90 & 15.60 & 13.70 \\
$\beta$-ala & 4.50 & 2.95 & 1.38 & 2.38 & 0.40 & 15.50 & 15.10 \\
Ala & 11.89 & 9.78 & 3.41 & 15.07 & 2.10 & 67.70 & 65.60 \\
Val & 10.20 & 8.77 & 2.15 & 6.49 & 1.50 & 53.30 & 51.80 \\
Phe & 26.83 & 31.05 & 1.73 & 2.39 & 1.40 & 132.40 & 131.00 \\
Leu & 7.63 & 7.29 & 2.36 & 6.17 & 1.00 & 37.50 & 36.50
\end{tabular}

a Abbreviations: S, sucrose; G, glucose; F, fructose; So, sorbitol; $P$, polyphenols; WSP, water-soluble pectin; CSP, chelator-soluble pectin; HASP, hydrochloric acid-soluble pectin; Q, quinic; M, malic; Sh*, shikimic (mg/kg); Asp, aspartic; Glu, glutamic; Asn, asparagine; Ser, serine; Gln, glutamine; Gly, glycine; Arg, arginine; $\beta$-Ala, $\beta$-alanine; Ala, alanine; Val, valine; Phe, phenylalanine; Leu, leucine; concentration of amino acids in $\mathrm{mg} / \mathrm{kg}$.

and Partial Least Squares (PLS). PCA allows us to reduce the dimensionality of the data matrix and to ascertain the relationship between the original variables and the factorial axes which are a linear combination of the original variables. The LDA method permits us to construct a linear combination of the original variables (canonical variable) in order to classify the samples belonging to unripe and ripe categories. The mathematical rule computed is used to predict the ripening stage of unknown cider apples. The PLS algorithm was used to establish a regression model between two criterion variables, a binary response $Y(Y=0$ for unripe apples and $Y=1$ for ripe apples) and an ordinal variable (picking order), and 23 predictor variables (chemical markers of ripening). The chemical variables were autoscaled before statistical treatment. The range of picking order was unified; the new variable $\left(Z_{i j}\right)$ was defined as $Z_{i j}: X_{i j}-\min X_{i j} / \max X_{i j}-\min$ $\mathrm{X}_{\mathrm{ij}}$.

\section{RESULTS AND DISCUSSION}

Univariate Analysis. Table 1 lists the mean values and other descriptive statistics for each chemical variable monitored during the ripening process of apple cider cultivars. Before multivariate data treatment was employed, a univariate analysis was carried out using F isher's test. The most discriminatory variables were fructose and phenylalanine (Fisher's weights: 0.67 and $0.64, p<1 \%$, respectively). However, the use of the most discriminatory variable, namely fructose, did not allow us to differentiate between both classes (unripe and ripe), so multivariate treatment of the data was needed.

Factor Analysis of the Internal Structure. Seven factors, which accounted for $75 \%$ of the variance, were considered using Kaiser's criterion (eigenvalues > 1.0 were chosen). Pectins, the chelator-soluble pectin (CSP), the water-soluble pectin (WSP), and the hydrochloric acid-soluble pectin (HASP), sorbitol, and certain amino acids such as serine, glycine, arginine, $\beta$-alanine, valine, and leucine were closely correlated to the first factor 


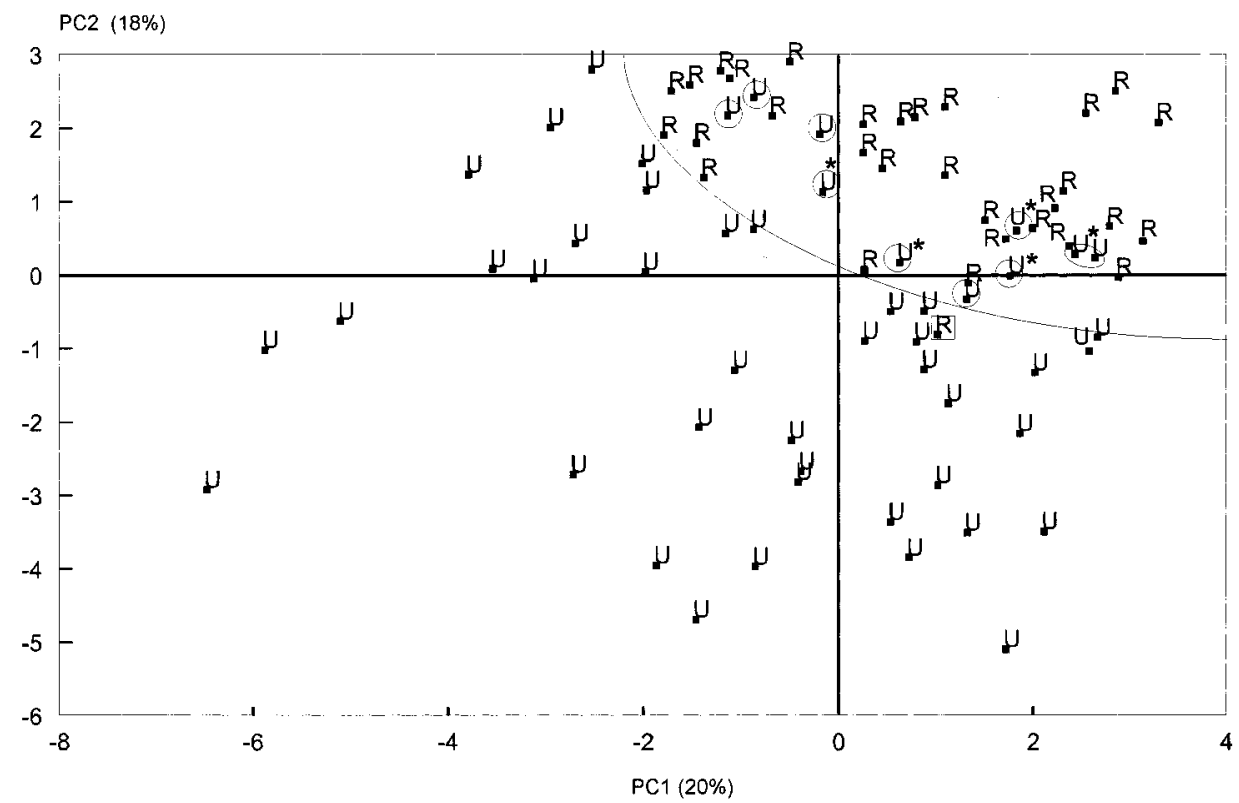

Figure 1. Two factorial axes projection of apples. U, unripe class; R, ripe class. (*) Corresponds to the samples classified initially as unripe and that are on the borderline between ripe and unripe categories.

(pectins and sorbitol show positive correlation and amino acids, negative correl ation). In contrast, sugars, such as fructose and glucose, polyphenols, malic, and quinic acids, and amino acids such as glutamic acid and phenylalanine were correlated to the second principal component (sugars presents a positive correlation and the remaining analytes, a negative correl ation). Figure 1 displays the projection of samples onto the plane formed by the first two principal components (PC). A data structurization can be visualized from this figure since the ripe apples are situated in the upper righthand corner of the map, which corresponds to samples with high levels of sugars and pectins and low levels of quinic and malic acids, polyphenols, and amino acids. As is well-known, in the final stages of fruit senescence, the concentration of sugars and pectins increases as a consequence of the metabolization of starch and malic acid and the degradation of structural polysaccharides, while quinic acid decreases probably due to the synthesis of phenolic acids. Likewise, in these stages of fruit ontogeny, the level of amino acids generally decreases, probably due to the increase in proteosynthetic activity of the fruit.

The data structurization visualized by PCA shows that the usual ripening classification criterion of cider apple cultivars, which is based on the estimation of certain global parameters such as starch, acidity, and total sugars, was not adequate for establishing the ripening stage of the fruit. For instance, some samples (* in Figure 1), classified initially as unripe apples, and that are on the borderline between unripe and ripe classes, were placed in the map zone corresponding to ripe fruits (Figure 1 ).

Linear Discriminant Analysis (LDA). Each original variable has an associated loading that shows its importance in the prediction capacity of the LDA method. Twelve of the 23 original variables, namely fructose, glucose, sucrose, sorbitol, chelator-sol uble pectin, water-soluble pectin, hydrochloric acid-soluble pectin, malic acid, glycine, serine, valine, and glutamic acid, had loadings higher than $|0.25|$, hence these variables were selected to construct the mathematical procedure for classifying apple cultivars on the basis of their
Table 2. Classification Matrix for LDA Method

\begin{tabular}{cccc}
\hline & \multicolumn{3}{c}{ assigned category } \\
\cline { 2 - 4 } true category & $\mathrm{U}$ & $\mathrm{R}$ & hits (\%) \\
\hline $\mathrm{U}$ & 45 & 4 & 91.8 \\
$\mathrm{R}$ & 2 & 29 & 93.6 \\
overall & & & 92.5
\end{tabular}

Table 3. Prediction Matrix for LDA Method Validation

\begin{tabular}{crrc}
\hline & \multicolumn{3}{c}{ assigned category } \\
\cline { 2 - 4 } true category & $\mathrm{U}$ & $\mathrm{R}$ & hits (\%) \\
\hline $\mathrm{U}$ & 42 & 7 & 85.7 \\
$\mathrm{R}$ & 5 & 26 & 83.9 \\
overall & & & 85.0
\end{tabular}

degree of ripening. All the variables sel ected by LDA, with the exception of sucrose, were correlated with the two first factorial axes computed by PCA. As we can see in Table 2, the classification capacity was higher than $92 \%$ (the majority of samples misclassified are on the borderline between ripe-unripe classes), and the prediction capacity (Table 3), estimated by cross-validation using three groups for cancellation, was $85 \%$. In consequence, we can consider that the mathematical procedure computed is sufficiently satisfactory for discriminating apple cultivars on the basis of their degree of ripening.

The influence of the ripening process on the chemical composition of fruits can be shown from the correlation matrix computed between the chemical variables and the binary response ( $Y$ ) and picking order (Table 4). As was expected, $Y$ and the picking order variables were closely correlated (0.75). The chemical variables with a correlation coefficient higher than $\mid 0.25$ in relation to the picking order were sugars, polyphenols, malic and quinic acids, and the following amino acids: glutamic acid, serine, glycine, arginine, and phenylalanine. As was expected, amino acids, polyphenols, and organic acids showed a negative correlation with the picking order variable, as their concentration decreased during ripening, while sugars showed a positive correlation with this variable, as their concentration increased during ripening. All chemical variables correlated with the picking order were in turn correlated with the two 
Table 4. Correlation Matrix for 23 Chemical Variables, the Binary Response ( $Y$ ), and the Order of Picking

\begin{tabular}{llllllllllll}
\hline Y & 1.00 & & & & & & & & & & \\
Pick & 0.75 & 1.00 & & & & & & & & \\
S & 0.36 & 0.59 & 1.00 & & & & & & & \\
G & 0.38 & 0.26 & -0.25 & 1.00 & & & & & & \\
F & 0.50 & 0.38 & 0.14 & 0.50 & 1.00 & & & & & \\
So & 0.45 & 0.47 & 0.57 & 0.00 & 0.09 & 1.00 & & & & \\
P & -0.19 & -0.30 & 0.15 & -0.63 & -0.07 & -0.06 & 1.00 & & & & \\
WSP & 0.00 & -0.07 & 0.02 & -0.39 & -0.02 & 0.13 & 0.44 & 1.00 & & & \\
CSP & 0.31 & 0.18 & 0.22 & -0.29 & 0.00 & 0.44 & 0.23 & 0.60 & 1.00 & & \\
HASP & 0.04 & -0.12 & 0.05 & -0.42 & -0.26 & 0.39 & 0.32 & 0.44 & 0.60 & 1.00 & \\
Q & -0.40 & -0.57 & -0.36 & -0.37 & -0.47 & -0.17 & 0.44 & 0.15 & 0.04 & 0.34 & 1.00 \\
M & -0.25 & -0.27 & -0.04 & -0.37 & -0.54 & -0.07 & 0.27 & 0.17 & 0.16 & 0.37 & 0.36 \\
Sh & -0.16 & -0.16 & -0.34 & 0.00 & -0.38 & 0.10 & -0.17 & -0.10 & -0.09 & 0.17 & 0.47 \\
Asp & -0.20 & -0.05 & 0.04 & -0.09 & -0.14 & -0.10 & -0.02 & -0.18 & -0.35 & -0.13 & -0.07 \\
Glu & -0.43 & -0.35 & 0.00 & -0.48 & -0.63 & 0.16 & 0.08 & 0.07 & 0.03 & 0.43 & 0.32 \\
Asn & -0.15 & -0.24 & -0.14 & 0.15 & -0.03 & -0.33 & 0.07 & -0.21 & -0.32 & -0.25 & 0.04 \\
Ser & -0.33 & -0.35 & 0.00 & -0.03 & 0.11 & -0.20 & 0.05 & -0.24 & -0.28 & -0.23 & 0.03 \\
GIn & -0.03 & 0.06 & 0.31 & -0.25 & -0.09 & 0.08 & 0.20 & 0.06 & 0.09 & 0.11 & 0.13 \\
Gly & -0.30 & -0.28 & -0.06 & -0.26 & -0.17 & -0.35 & 0.16 & -0.15 & -0.34 & -0.11 & 0.13 \\
Arg & -0.37 & -0.37 & 0.08 & -0.37 & -0.30 & -0.25 & 0.28 & -0.08 & -0.25 & -0.06 & 0.15 \\
$\beta$-ala & -0.26 & -0.24 & 0.12 & -0.24 & -0.05 & -0.37 & 0.23 & -0.16 & -0.32 & -0.32 & -0.03 \\
Ala & 0.00 & -0.04 & 0.45 & -0.10 & -0.07 & 0.29 & 0.09 & -0.06 & -0.03 & 0.05 & 0.00 \\
Val & -0.29 & -0.24 & -0.07 & 0.22 & -0.06 & -0.23 & -0.22 & -0.44 & -0.53 & -0.51 & -0.04 \\
Phe & -0.45 & -0.55 & -0.41 & -0.17 & -0.53 & -0.25 & 0.06 & -0.01 & -0.17 & 0.09 & 0.66 \\
Leu & -0.16 & -0.16 & -0.03 & 0.04 & 0.11 & -0.21 & -0.02 & -0.25 & -0.38 & -0.28 & -0.11 \\
& $\mathrm{Y}$ & $\mathrm{Pick}$ & $\mathrm{S}$ & $\mathrm{G}$ & $\mathrm{F}$ & $\mathrm{So}$ & $\mathrm{P}$ & $\mathrm{WSP}$ & $\mathrm{CSP}$ & $\mathrm{HASP}$ & $\mathrm{Q}$
\end{tabular}

$\begin{array}{llll}\text { M } & 1.00 & & \\ \text { Sh } & 0.09 & 1.00 & \\ \text { Asp } & -0.19 & 0.20 & 1.00\end{array}$

Asp $\quad-0.19 \quad 0.20 \quad 1.00$

$\begin{array}{llll}\text { Glu } & 0.14 & 0.36 & 0.50\end{array}$

Asn $\quad 0.13 \quad-0.18 \quad 0.12$

$\begin{array}{llll}\text { Ser } & -0.03 & -0.08 & 0.15\end{array}$

$\begin{array}{llll}\text { GIn } & 0.14 & -0.06 & 0.14\end{array}$

Gly $\quad 0.25 \quad-0.04 \quad 0.25$

$\begin{array}{llll}\text { Arg } & 0.39 & -0.22 & 0.09\end{array}$

$\begin{array}{llll}\beta \text {-ala } & 0.13 & -0.41 & 0.04\end{array}$

$\begin{array}{llll}\text { Ala } & -0.07 & -0.06 & 0.13 \\ \text { Val } & -0.41 & -0.01 & 0.46\end{array}$

$\begin{array}{llll}\text { Val } & -0.41 & -0.01 & 0.46 \\ \text { Phe } & 0.20 & 0.46 & 0.16\end{array}$

$\begin{array}{llll}\text { Leu } & -0.25 & -0.19 & 0.16\end{array}$

$\begin{array}{lll}1.00 & & \\ -0.09 & 1.00 & \\ 0.03 & 0.25 & 1.00 \\ 0.19 & -0.01 & 0.29 \\ 0.09 & 0.18 & 0.49 \\ 0.13 & 0.21 & 0.37 \\ -0.10 & 0.30 & 0.33 \\ 0.17 & 0.01 & 0.38 \\ 0.23 & 0.26 & 0.34 \\ 0.44 & 0.21 & 0.02 \\ -0.03 & 0.02 & 0.30 \\ \text { Glu } & \text { Asn } & \text { Ser }\end{array}$

$\begin{array}{ll}1.00 & \\ 0.22 & 1.00 \\ 0.24 & 0.64 \\ 0.13 & 0.42 \\ 0.24 & 0.25 \\ -0.04 & 0.14 \\ -0.10 & 0.06 \\ -0.03 & 0.31 \\ \text { Gln } & \text { Gly }\end{array}$
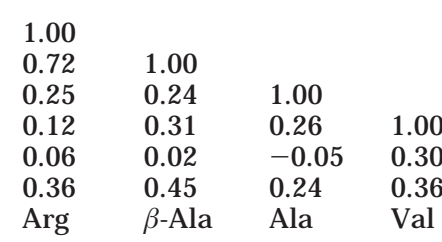

1.00

$\begin{array}{lll}.36 & 0.08 & 1.00\end{array}$

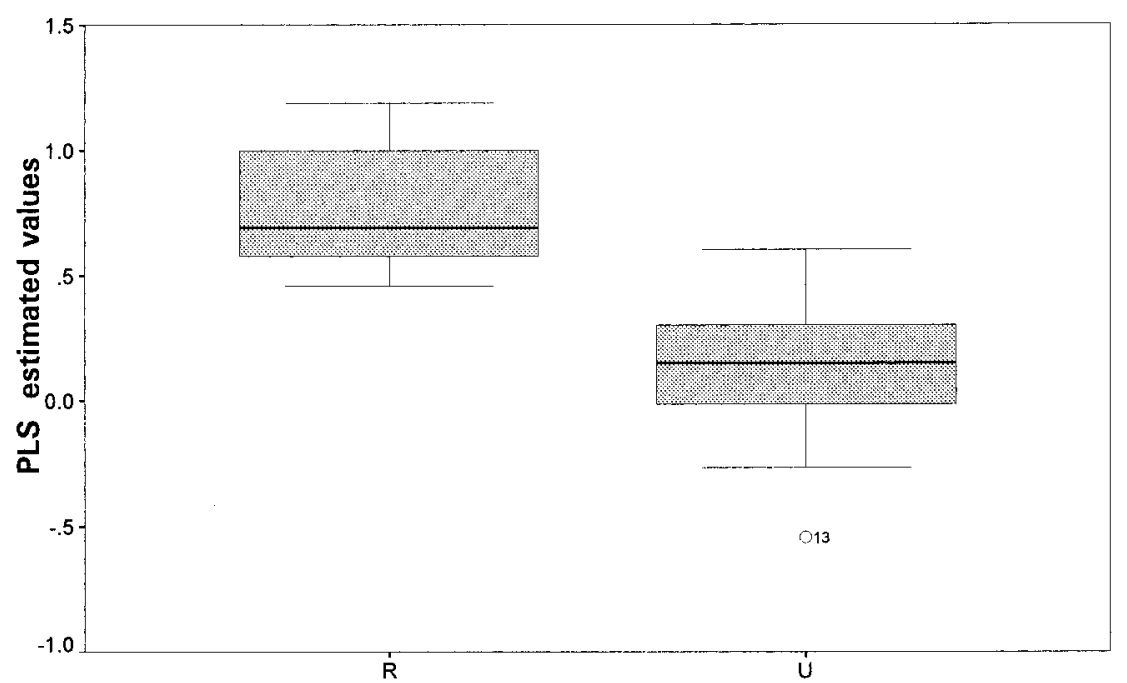

Figure 2. Multiple box-whisker plots for PLS computed values: $U$, unripe; R, ripe.

first factorial axes, and the majority of these variables were also the dominant ones in LDA analysis.

Modeling Methods. Partial Least Squares (PLS). The binary response $(Y)$ and the picking order were subjected to multivariate regression by PLS-2 using the chemical variables as predictors. A cross-validation method with three groups for cancellation was used to validate the model constructed. This model consisted of six latent variables (maximum cross-validated explained variance values, 69.7 and $89.3 \%$ for $Y$ and picking order variables, respectively); an explained variance (EV) of 75.8 and $91.0 \%$; and a multiple linear correlation coefficient of 77.8 and $91.7 \%\left(R^{2}\right)$ for $Y$ and picking order variables having been estimated, respectively. The most relevant variables in the PLS-2 model in order of importance were sugars (sucrose, glucose, and fructose), serine, shikimic acid, sorbitol, and hydrochloric acid-soluble pectin. Figure 2 shows a multiple box-whisker pl ot for PLS values computed with the PLS-2 model for the binary response $(Y)$; as can be seen, 
the differentiation of the categories (unripe and ripe) is satisfactory. Likewise, the picking time of apples chosen for cidermaking is highly critical due to the influence detected of the ripening process on the chemical markers studied ( $R^{2}$ picking order: 91.7\%), which, in turn, are closely related to the quality of the processed apple products.

\section{CONCLUSIONS}

Data obtained from HPLC and FIA analyses of the chemical variables (sugars, organic acids, amino acids, pectins, and polyphenols) involved in the process of fruit senescence together with chemometrics (PCA, LDA, and PLS-2) have allowed the authors to typify cider apple cultivars, categorized as ripe and unripe, according to their degree of ripening. Fructose was the most relevant variable for discriminating both classes, although this variable was not sufficient to differentiate unripe and ripe apples. The PCA method provided an adequate data structurization using only two dimensions, and a feasible mathematical decision rule was established with the use of the LDA method (prediction capacity = $85 \%$ ) in order to classify apples on the basis of their degree of ripening; the canonical variable computed was closely related to fructose, glucose, sucrose, sorbitol, pectins, malic acid, glycine, serine, valine, and glutamic acid. Likewise, a linear model computed by means of the PLS-2 algorithm allowed us to predict the degree of ripening of the apple cider varieties.

\section{ACKNOWLEDGMENT}

This work was made possible by financial support from the CICYT (ALI 96-1219-C02-01).

\section{LITERATURE CITED}

Ackermann, J .; Fischer, M.; Amadò, R. Changes in sugars, acids, and amino acids during ripening and storage of apples (Cv. Glockenapfel). J . Agric. Food Chem. 1992, 40, 11311134.

Berüter, J . Carbohydrate partitioning and changes in water relations of growing apple fruit. J . Plant Physi ol. 1989, 135, 583-587.

Blanco, D.; Gutiérrez, M. D.; Mangas, J . J .; Noval, A. Determination of sugars and alcohols in apple juice and cider by high performanceliquid chromatography. Chromatographia 1988a, 25, 701-706.

Blanco, D.; Morán, M. J .; Gutiérrez, M. D.; Mangas, J. J . Application of HPLC to the characterization and control of individual acids in apple extracts and ciders. Chromatographia 1988b, 25, 1054-1058.

Blanco, D.; Picinelli, L.; Gutiérrez, M. D.; Mangas, J. J. Determination of amino acids in apple extracts by high performance liquid chromatography. Chromatographia 1990, 29, 155-160.
Blanco, D.; Morán, M. J .; Gutiérrez, M. D.; Moreno, J .; Dapena, E.; Mangas, J . J . Biochemical study of the ripening of cider apple varieties. Z. Lebensm. Unters. Forsch. 1992a, 194, 3337.

Blanco, D.; Picinelli, L.; Gutiérrez, M. D.; Mangas, J.J. Determination of amino acids in ripening apples by high performance liquid chromatography. Z. Lebensm. Unters. Forsch. 1992b, 194, 134-138.

Cruz Ortiz, M.; Saez, J. A.; López Palacios, J. Typification of alcoholic distillates by multivariate techniques using data from chromatographic analyses. Analyst 1993, 118, 801805.

Fils-Lycaon, B.; Buret, M.; Drouet, A.; Hartmann, C.; Duprat, F. Ripening and overripening of cherry fruit: use of principal component analysis to check fruit picking and sampling method pertinence and to sel ect the most discriminant analysis criteria. Sci. Aliments 1988, 8, 383-396.

Forina, M.; Leardi, R.; Armanino, C.; Lanteri, S. In PARVUS. An Extendable Package of Programs for Data Exploration, Classification and Correlation; Elsevier: Amsterdam, 1988.

Henschke, P.; J iranek, V. Yeasts-metabolism of nitrogen compounds. In Wine Mi crobiol ogy and Bi otechnol ogy; Fleet, G. H., Ed.; Harwood Academic Publishers: Switzerland, 1993; Chapter 4.

Kintner, P.; Van Buren, J . Carbohydrate interference and its correction in pectin analysis using the MHDP. J . Food Sci 1982, 47, 756-764.

Lea, A. G. H. Cidermaking. In Fermented Beverage Production; Lea, A. G. H., Piggott, J. R., Eds.; Blackie Academic \& Professional: London, 1995; Chapter 4.

Le Quere, J. M.; Latreille, R.; Rouxel, B.; Drilleau, J. F. Prefermentation clarification of cider apple musts. Industr. Alim. Agr. 1988, Mars, 137-139.

Mangas, J. J .; Dapena, E.; Rodríguez, M. S.; Moreno, J .; Gutiérrez, M. D.; Blanco, D. Changes in pectic fractions during ripening of cider apples. HortSci. 1992, 27, 328-330.

Mangas, J .; Suárez, B.; Blanco, D. Automated determination of total polyphenols in apple juice. Z. Lebensm. Unters. Forsch. 1993, 197, 424-426.

Mangas, J .; Rodríguez, R.; Moreno, J .; Suárez, B.; Blanco, D. $E$ volution of aromatic and furanic congeners in the maturation of cider brandy: A contribution to its characterization. J . Agric. Food Chem. 1996, 44, 3303-3307.

Mangas, J .; Rodríguez, R.; Moreno, J .; Suárez, B.; Blanco, D. Furanic and phenolic composition of cider brandy. A chemometric study. J . Agric. F ood Chem. 1997, 45, 4076-4079.

McRae, K.; Lidster, P.; DeMarco, A.; Dick, A. Comparison of the polyphenol profiles of apple fruit cultivars by correspondence analysis. J. Sci. Food Agric. 1990, 50, 329342.

Richmond, M.; Brandao, S.; Gray, J .; Markakis, P.; Stine, C. Analysis of simple sugars and sorbitol by HPLC. J . Agric. F ood Chem. 1981, 29, 4-7.

Thibault, J. F. Automatisation du dosage des substances pectiques par la methode au MHDP. Lebensm.-Wiss. u.Technol. 1979, 12, 247-251.

Received for review J anuary 27, 1998. Revised manuscript received J une 11, 1998. Accepted J uly 7, 1998.

J F 980078N 